\title{
Design and experiment of electro hydraulic active suspension for controlling the rolling motion of spray boom
}

\author{
Longfei Cui ${ }^{1,2}$, Xinyu Xue ${ }^{1 *}$, Feixiang Le ${ }^{1}$, Hanping $\mathrm{MaO}^{2}$, Suming Ding ${ }^{1}$ \\ (1. Nanjing Research Institute for Agricultural Mechanization, Ministry of Agriculture and Rural Affairs, Nanjing 210014, China; \\ 2. Key Laboratory of Modern Agricultural Equipment and Technology, Ministry of Education, Jiangsu University, Zhenjiang 212013, China)
}

\begin{abstract}
Boom sprayer is one of the most commonly used plant protection machinery for spraying pesticide. Studies have shown that the efficiency of chemicals is highly correlated with the uniformity of spray distribution patterns. As the boom is a large and flexible structure, boom rolling leads to overlapping and leakage of the pesticides. In order to improve spray uniformity, the boom attitude should be kept parallel to the ground slope or to the crop canopy beneath the boom. Passive suspension can attenuate frequencies above its resonance frequency, but nothing can be done to align the boom to the sloping ground. Therefore, an active suspension system is designed, which includes DSP-based controller, a servo valve, a hydraulic cylinder, two ultrasonic sensors, one inertial attitude sensor, and the developed control procedures. In order to prevent the wrong response of the control system caused by the high frequency component due to uneven crop canopy or rough ground. A special signal processing algorithm was proposed, including the limiting filter, smoothing algorithm and data fusion algorithm based on optimal weight. The transient and steady-state performances of the boom control system using velocity feedforward PID algorithm were tested on a six DOF motion simulator. It can be seen that the low-frequency tracking performance of the boom was greatly improved after the electro-hydraulic active suspension was added. At the resonance frequency, the peak angle of active suspension and passive suspensions are $0.72^{\circ}$ and $1.29^{\circ}$ respectively, and the resonance peak is greatly reduced. The controller was implemented on a self-propelled boom sprayer and validated under field conditions, the standard deviation of the roll angle of the boom with active suspension is $0.40^{\circ}$, compared with $1.04^{\circ}$ of the sprayer chassis. Experimental results show that the active suspension control system can effectively reduce the effect of ground excitation disturbance on the application process, and has good tracking performance for low frequency terrain change. Keywords: boom sprayer, active suspension, electro-hydraulic servo system, six DOF motion simulator, signal process, motion control
\end{abstract}

DOI: $10.25165 /$ j.ijabe.20191204.4648

Citation: Cui L F, Xue X Y, Le F X, Mao H P, Ding S M. Design and experiment of electro hydraulic active suspension for controlling the rolling motion of spray boom. Int J Agric \& Biol Eng, 2019; 12(4): 72-81.

\section{Introduction}

With the continuous growth of the world population, agricultural productivity will continue to increase. It is estimated that agricultural productivity will increase by $60 \%$ by $2050^{[1]}$. A major factor limiting yield is weed control and pest control. The boom sprayers are widely used in crop protection on the fields against diseases, weeds, pathogens, and others ${ }^{[2,3]}$. The width of the mainstream boom sprayer in China is about 10-24 m, unwanted boom motions typically occur when sprayer tires go over uneven terrain field surfaces ${ }^{[4,5]}$. Spray boom motions, mainly vertically rolling has a dramatic effect on the spray distribution pattern ${ }^{[6,7]}$. It is pointed out that the efficiency of chemical pesticides is highly correlated with the uniformity of the spray distribution pattern ${ }^{[8]}$.

Received date: 2018-09-11 Accepted date: 2019-06-30

Biographies: Longfei Cui, PhD, Assistant Research Fellow, research interests: agricultural machinery system dynamics and control technology, Email: cuilong.fei@163.com; Feixiang Le, research probationer, research interest: vehicle engineering, Email: 469579103@qq.com; Hanping Mao, PhD, Professor, Research interests: agricultural equipment and engineering, Email: mhp@ujs.edu.cn; Suming Ding, Research fellow, research interest: crop protection and machinery engineering,Email: dsmchina@sina.com.

*Corresponding author: Xinyu Xue, PhD, Research fellow, research interest: crop protection and machinery engineering, Nanjing Research Institute for Agricultural Mechanization, Ministry of Agriculture, Nanjing 210014, China. Tel: +86-25-84346243, Email: xuexynj@qq.com.
Studies point out that boom roll is responsible for variations in spray deposit ranging from 0 to $1000 \%(100 \% \text { is ideal })^{[9,10]}$. So, this paper mainly focuses on the roll motion of the boom, the effect on spray distribution of which is shown in Figure 1.

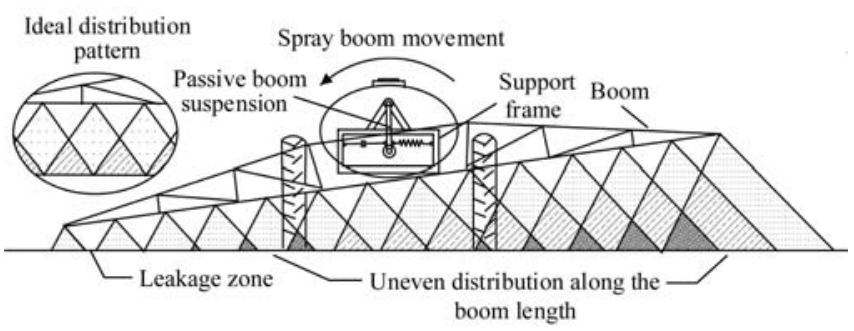

Figure 1 Movement of the spray boom and the droplet distribution

The spray boom will parallel to the ground by using active suspension, it will give a more even coverage of spray and can also be mounted closer to the crop without fear of the boom tips striking the ground. The lower the boom, the less the spray is affected by the wind and so drift is reduced ${ }^{[11-13]}$. However, the boom sprayers in China usually have no suspension or using passive suspension. The common passive suspension includes pendulum suspension, trapezoidal suspension, twin universal link suspension, etc. Research shows that the passive suspension can satisfy the high frequency requirement, but cannot meet the low frequency requirement, for example, align the boom to ground 
undulations $^{[14-17]}$. The development of active boom suspension restricts the improvement of the spraying effectiveness and quality of the boom sprayer in China ${ }^{[2,4]}$.

Several investigators have studied a number of boom suspension techniques, both theoretically and experimentally ${ }^{[19-22]}$. In these works, different control methods such as proportional (P), proportional-integral (PI) and $\mathrm{H} \infty$ control theory have been tried to control the motion of boom ${ }^{[23-27]}$. In developed countries, automatic boom control systems were developed, such as Norca UC5, Amazon AMAPAD and Mueller TOUCH800. It is based on ultrasonic sensors for measuring the distance to the field or the canopy of the plants and a heuristic controller for the leveling actions. These systems can ensure satisfactory performance at moderate speeds in large plains with flat terrain and large areas of cultivated land, but have problems at higher speeds in hills and mountains ${ }^{[23,24]}$. However, the cultivated land resources in China are mainly hilly and mountainous areas, and the planting area is dispersed. The complex farmland pavement environment requires high response speed of the electro-hydraulic servo system of the active boom suspension. Moreover, these commercial controllers have special requirements for the machine configuration and hydraulic system design of the suspension. Therefore, none of these commercial controllers has been used by the sprayers made in China. Most spraying machines are not equipped with boom suspension, only a few manufacturers develop appropriate control systems independently.

In the development of the boom suspension control system, Chen et al. ${ }^{[28]}$ designed a $12 \mathrm{~m}$ spray boom angle control system based on AT89S52 MCU. Wang et al. ${ }^{[29,30]}$ used Siemens S7-200 PLC controller to realize the angle control of the spray boom. The common control algorithm of boom suspension is mainly based on the PID method. Because of the limitation of internal compiler and programming language, PLC controller is not suitable for writing and applying complex control algorithm directly. MCU is suitable for simple measurement and control system. It is too slow for complex signals processing algorithm to be suitable for motion control of a large spray boom. In recent years, DSP chips have been widely used in the field of industrial control because of high speed, flexibility, programmability and low power consumption. Therefore, in this paper, a complete DSP hardware circuit, and control software are developed, the speed feed-forward compensation PID control method is used to achieve high-precision control of the spray boom.

Another noticeable problem in the design of control systems is the processing of sensor signals. The height of each proximity sensor to the crop canopy will have low frequency signal components due to a change in the ground slope; in addition, the noise components are added due to the bumpy ground or the uneven crop canopy below the sensor. These noise components seriously affect the accuracy of the control system. Therefore, in this paper, we focus on the design of filtering algorithms such as limiting the amplitude and dynamic smoothing of sampling signals. Then, data fusion of pre-processed ultrasonic signal and inclination sensor was carried out. Finally, the fused data was used as the input of the control algorithm to improve the measurement accuracy.

The main objective of this research is to design and validate the performance of DSP-based controller and the proposed signal processing algorithm for the active boom suspension. The developed DSP control terminal and human-machine interface are matched with a $28 \mathrm{~m}$ large pendulum boom suspension.
Laboratory tests are carried out under various test conditions by using a six-degree-of-freedom motion simulator. The final controller was implemented on the 3WPHS-600A self-propelled sprayer. The reliability of the active suspension control system is verified by the experiment in the paddy field.

\section{Pendulum boom suspension configuration and working principle}

\subsection{Suspension configurations}

A boom suspension system is provided for controlling a roll position of a boom rotatably coupled to a support frame, the boom comprising left and right wing sections (where the rolling of the boom refers to a clockwise or counterclockwise rotation of the boom relative to the support frame). An active actuator is configured for rotating the boom relative to the support frame in response to a roll control signal. Figure 2 shows a plan view of a boom with an active suspension, whose length is $28 \mathrm{~m}$, and weight is $926 \mathrm{~kg}$. The boom is mounted on a sprayer via the active suspension. The suspension consists of a support frame 1, a pendulum rod 2, hydraulic actuator3, center boom frame 4, bracket 5 , vertical shock absorber 6 and lateral shock absorber 7 .

The support frame 1 is used to bear the gravity of the boom system and the inertia load. The pendulum rod 2 is jointed with the support frame 1 in rotation center $O$ through a revolute joint. The left side and right side of boom arms are connected with the center boom frame 4 by the revolute pairs. The center boom frame 4 is coupled to the pendulum rod 2 to permit clockwise or counterclockwise rotation around the point $P$. the pendulum $\operatorname{rod} 2$ is connected to the boom support frame 4 with a roll hydraulic cylinder 3. As the roll cylinder 3 extends (or retracts) it causes the center boom frame 4 to rotate in a counterclockwise (or clockwise) direction. This exerts a torque on the center boom frame, in turn, this causes the entire boom framework to rotate a new roll position is established. The bracket 5 is mounted at the end of the pendulum rod 2. Vertical shock absorbers are articulated between the center frame 3 and the bracket 4 , to attenuate vibration in the vertical direction. Lateral shock absorber 7 is connected between center frame 4 and the support frame 1.

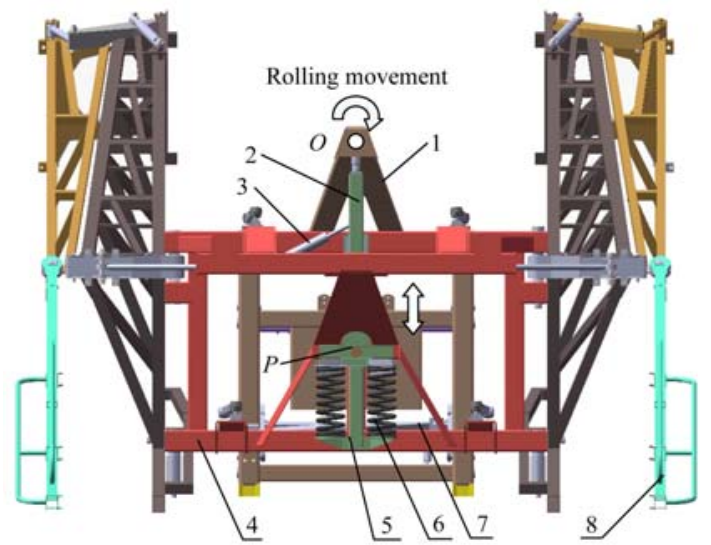

1. Support frame 2. Pendulum rod 3. Hydraulic cylinder 4. Center boom frame 5. Bracket 6. Vertical shock absorber 7. Lateral shock absorber 8. Spray boom

Note: $O$ is rotation center of the pendulum rod; $P$ is rotation center of the center frame.

Figure 2 Structure of spray boom and pendulum suspension

The active suspension consists of an external power source, hydraulic actuator, non-contact proximity transducers and signal processing, feedback and control elements. The oil power source is supplied by the pump of the chassis. The proximity transducers 
monitor boom to ground height. This information is processed by a DSP controller which filters out high frequency noise and then supplies the appropriate command signal to the actuator, causing it to extend or retract at a rate related to the difference between the ground slope and boom attitude.

\subsection{Design of hydraulic circuit for boom suspension}

Schematic diagram of the hydraulic system as shown in Figure 3 , there are 5 sections of the boom, consists of two inner booms, two outer booms and center boom frame, cylinder 4 and cylinder 5 are used to drive folding or unfolding of inner spray booms, cylinder 6 is configured for rotating the boom relative to the support frame. Cylinder 9 and cylinder 10 are used to drive folding or unfolding of outer spray booms. The one-way speed regulating valve 3 can be used to regulate the movement speed of the boom, so as to avoid the excessive speed and cause the boom oscillation. The liquid controlled one-way valve 2 plays the role of locking the oil road, When the sprayer stops working, the hydraulic system unloads, and the piston rod of the hydraulic cylinder stops moving so that the spray rod maintains a fixed attitude. Two-way directional valves control the motion of the single acting cylinder 7 and the adjustment of the height of the whole boom. Hydraulic tubing connects the clueless chamber of the hydraulic cylinder, and the reverse movement is driven by the gravity of the boom. The motion of the cylinder 6 is controlled by three positions four-way direct drive servo valve.

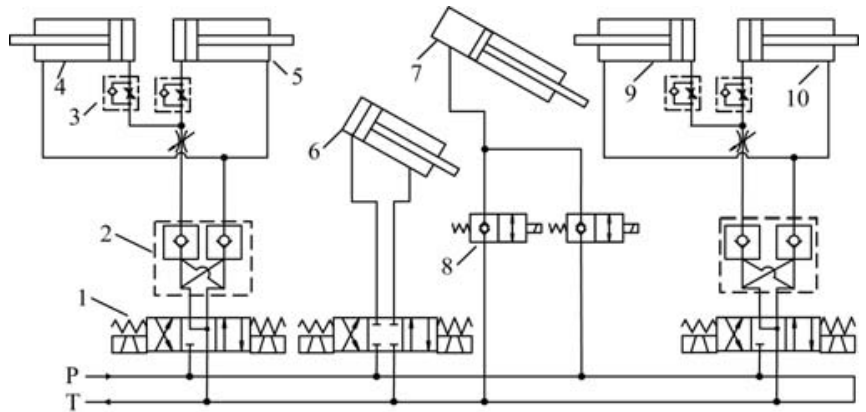

1. Three positions four-way reversing valve 2. Liquid control one-way valve 3. One-way valve 4,5. Cylinders for folding and unfolding of the inner boom 6. Cylinder for rolling control of the whole boom 7. Height adjustment cylinder 8. Two-way direction control valves 9,10 . Cylinders for folding and unfolding of the outer boom

Figure 3 Schematic diagram of the hydraulic system

\section{Design of electro-hydraulic active boom suspension}

\subsection{Performance specifications of boom suspension}

Boom suspension tries to keep the boom at right angles to gravity by isolating the boom from vibrations of the vehicle, induced by soil unevenness. An extensive study has shown that the transfer functions of the different vertical boom suspension, relating the output of the actuator which is used for leveling the boom, to the rolling boom motions, are similar in shape ${ }^{[23,24]}$.

The frequency-domain response characteristics of the boom suspension system are shown in the Figure 4. As can be seen, the high-frequency vibrations of the sprayer vehicle are suppressed very well, contrary to the low frequencies especially at the resonance frequency of the suspension where disturbances are even magnified.

The aim of this paper is to develop an electro-hydraulic active boom suspension which is able to follow the slopes of the field and reduce the resonance peak of the suspension.

The distinction between high and low frequencies depends on the speed of the spray vehicle, and the topography of the field. If, for example, the boom is to follow field undulations of wavelengths greater than $\lambda_{\min }$, at the maximum speed of $v_{\max }$, the suspension should transmit to the boom frequencies less than $f_{\max }$, and attenuate higher frequencies, where

$$
f_{\max }=\frac{v_{\max }}{\lambda_{\min }}
$$

Usually, the maximum speed of the self-propelled sprayer is $4 \mathrm{~m} / \mathrm{s}$, and the boom should follow the undulations of the wavelength greater than $40 \mathrm{~m}$. Therefore, from Equation (1), $f_{\max }$ equals to $0.1 \mathrm{~Hz}$. Since following the slope is a very slow motion and the passive suspension works adequately beyond its natural frequency $w_{\mathrm{n}}$, an active system intervening only below $0.1 \mathrm{~Hz}$ will provide the low-frequency motions of the boom without needing a lot of power.

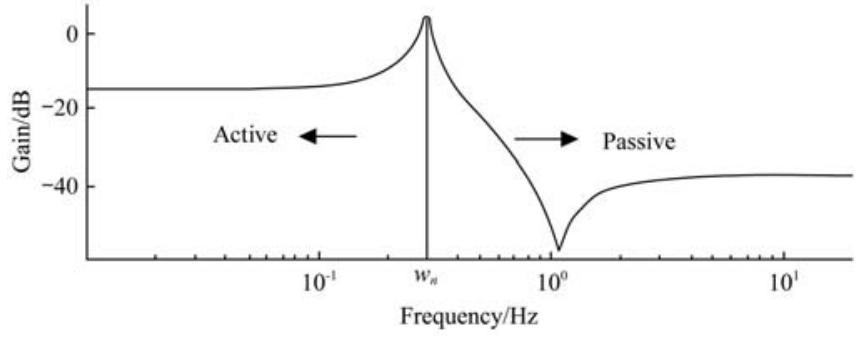

Figure 4 Active and passive working area of the suspension

\subsection{Hardware components of the servo system}

Figure 5 shows the schematic diagram of the hydraulic boom roll control system hardware components. The controller receives and processes measurement information signals from the sensors and produces therefrom a roll control signal configured to drive the valve within valve bank which operates the roll hydraulic cylinder, to control rolling of the boom and thus, in turn, assist in controlling the height of the boom along its length over the spray target.

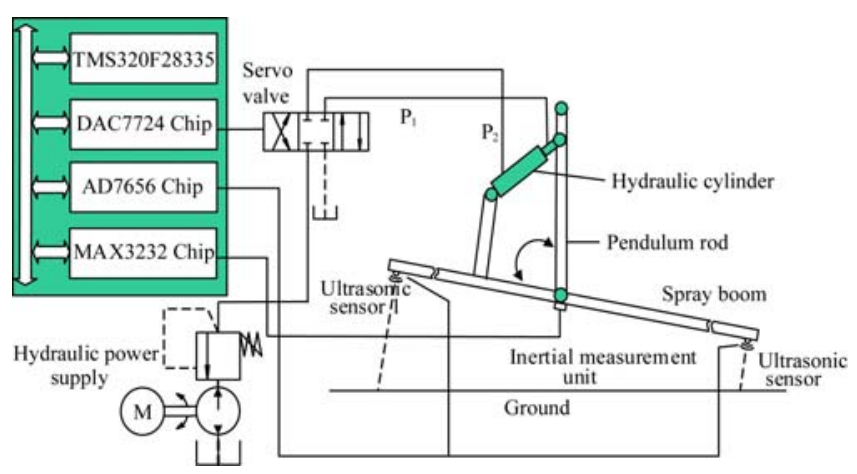

Figure 5 Schematic diagram of hydraulic servo control system hardware

The roll control system is composed of the distance measurement devices mounted as shown and comprising two ultrasonic sensors (U45Q, Bonner, Minnesota, USA), an inertial measurement unit (Ellipse-D-G4A2-B1, SBG, Cherbourg, France), and a controller in the form of a microprocessor (TMS320F28335, Texas Instruments, Texas, USA), a roll hydraulic cylinder and its associated servo valve (D633-308B, Moog, New York, USA) besides, a power source. The two ultrasonic sensors are mounted on the left and right sides of the boom respectively. The inertial sensor is installed in the middle position of the center boom. The output frequency of the ultrasonic sensor is $12.5 \mathrm{~Hz}$, the detection range is $250-3000 \mathrm{~mm}$. The output frequency of the inertial measurement unit is $200 \mathrm{~Hz}$, and the rolling angle measurement accuracy is $0.05^{\circ}$.

The supply pressure of the hydraulic station is $10 \mathrm{MPa}$, the 
single rod hydraulic cylinder is selected as an actuator, whose piston diameter is $40 \mathrm{~mm}$, the piston rod diameter is $20 \mathrm{~mm}$, and the stroke is $180 \mathrm{~mm}$. The servo valve (Moog D633-308B whose flow rate is $20 \mathrm{~L} / \mathrm{min}$ at 70 bar drops) whose bandwidth is above $50 \mathrm{~Hz}$ is used a power control element. The sampling time of the control system is $5 \mathrm{~ms}$, and this sample rate is both for data acquisition and the closed loop execution.

The hardware circuit of the control system is mainly composed of a digital signal processor (DSP), an A/D conversion circuit, a $\mathrm{D} / \mathrm{A}$ conversion circuit, a signal conditioning circuit and a communication circuit. In order to ensure the real-time and stability of the hydraulic servo control system, we choose the TMS320F28335 digital signal processor of Texas Instruments as the core of the system control, whose clock cycle is $150 \mathrm{MHz}$. As the control signals of the sensor feedback signal and servo valve are analog voltage signals, the control circuit should also include the A/D analog acquisition module (AD7656, ADI, Massachusetts, USA) and the D/A drive module (DAC7724, Texas Instruments, Texas, USA). After collecting the voltage output signal of the ultrasonic sensors, the data acquisition module converts the signal into digital signal that can be operated, then the digital control signal output by the controller is transformed into a voltage command signal through the $\mathrm{D} / \mathrm{A}$ module. When the servo proportional valve receives the control command, the hydraulic cylinder moves to the desired position. In addition, the communication circuit with the upper computer and the angle data acquisition circuit of the inertial sensor is designed by using the RS232 interface.

\subsection{Program development of control system}

\subsubsection{Main program of the control system}

The main function of the control system software is to collect and process the signal of the sensor, to calculate and output the control command of the servo valve, and to realize the input of the working parameters and the display of the working state of the system. The main program flow of the controller is shown in Figure 6.

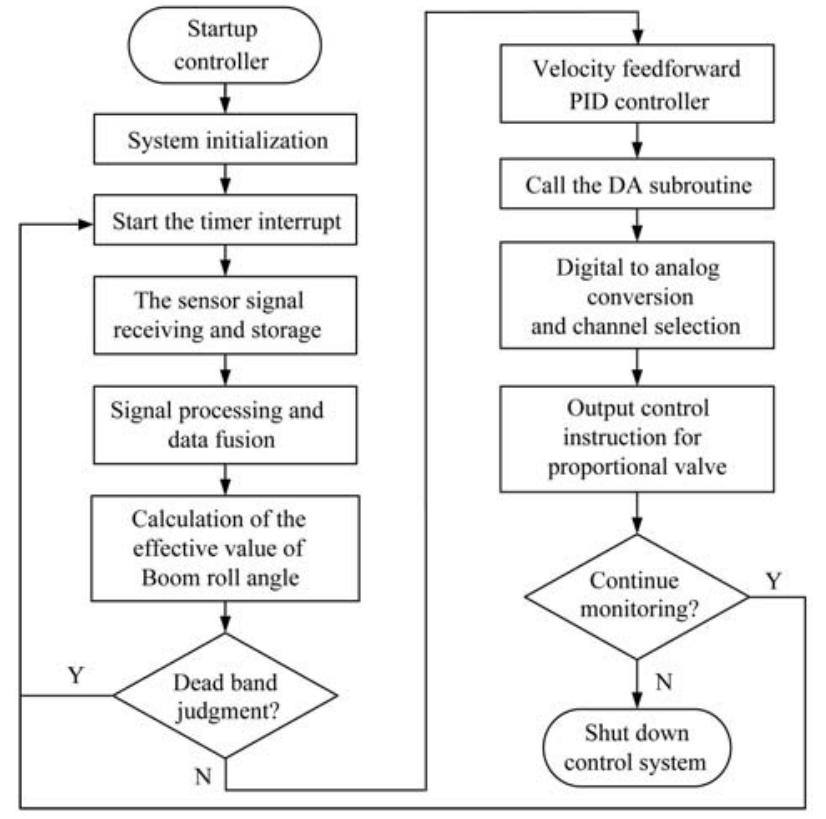

Figure 6 Flow chart of the main program

After the control system is started, it is automatically initialized. When the parameters are normal, a timer interrupt is executed. The controller unit continuously receives transmitted signals data from each ultrasonic sensors and inertial measurement sensor, then record into its memory. The processed signals were used as control input, the controller compares the control error to a dead band value and sets the boom roll control error value to zero when the comparison identifies that the boom roll control error value is less than the dead band value. Whereas the control error value is greater than the dead zone, the controller calculates the control command according to the control input.

3.3.2 Signal processing method

a) Design of limiting filter

Crops need to applicate pesticides at different growth stages, such as seedling stage, growth stage and flowering stage.

For example, the control of wheat powdery mildew at the growth period, cotton sprayed with defoliant at flowering stage, at this time the growth density of the spraying crops is relatively large, we usually choose crop height feedback control mode. Controlling the sprayer boom so that it maintains a fixed distance from the top of the crop can result in a situation in which, in the absence of crops under the sprayer boom, the control system will mistakenly consider that the ultrasonic pulse reflected from the ground beneath the sensor to be a reflection from the top of the crop which did not exist because it is the first echo received.

If the sprayer boom is being controlled such that a fixed distance between the top of the crop and the sprayer boom is being maintained then this erroneous interpretation of the reflection from the soil as a top of crop signal will cause the sprayer boom to be lowered to maintain the desired distance. However, the distance, in this case, would be between the sprayer boom and the ground level which has been erroneously interpreted as corresponding to the top of the crop.

Therefore, a limiting filtering algorithm for the height signal collected by ultrasonic sensors is proposed. Before the start of the control system, the average height of the crop $h$ and the desired distance between the boom tips and crop top $s$ should be set through the upper computer interface. The average crop height $h$ can be obtained by means of multi-point measurement prior to application.

As shown in Figure 7, when the sprayer passes through the area $A B$ in which there is no crop under any of the ultrasonic sensors. In this case, the control system receives a reflection of the ultrasonic pulse from the ground level $g$, instead of distance values for the top of crop distance value $c$. Fortunately, the controller does have an average crop top height value stored in its memory. If the error between the current distance and the previously measured distance is greater than the average crop height $h$, the current measurement is considered invalid. In addition, if in the following $N$ sampling periods, the errors are all greater than $h$, the controller determines a virtual top of crop distance $c^{\prime}$ by subtracting the average crop height value from the local ground distance value $g$ determined at the region between $A$ and $B$.

$$
c^{\prime}=g-h
$$

The controller then maintains the separation distance $s$ from the average height of the top layer of the crop. When the boom moves to position $B$, the ultrasonic sensors will receive a true echo from the top of the crop again and will send to the controller unit local values of top of crop distance. The controller unit will thus once raise or lower the boom again to maintain the separation distance $s$ between the boom and the top of the crop but this time the top of crop distance will be used for the top of crop level because it is available. 


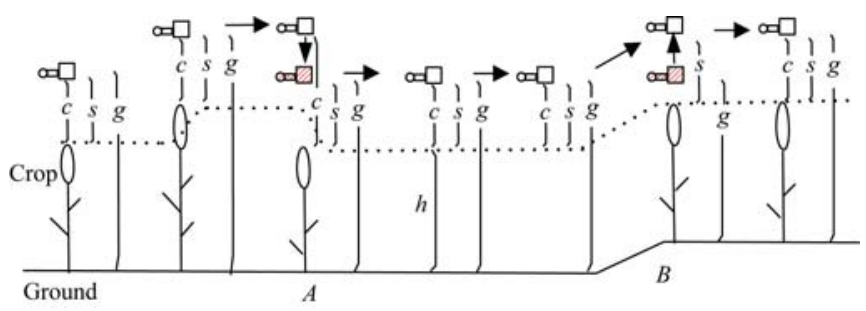

Note: $c$ represents the distance from the top of the crop; $s$ represents the desired distance from the crop top; $g$ represents the local ground distance value; $h$ represents the average height of the crop.

Figure 7 Limiting filtering algorithm based on the average height of the crop

In the same way, the distribution of crops is sparse in the seedling stage, sprayers usually use ground height feedback control mode for application, as described in detail above, and each ultrasonic sensor module communicates its local ground level distance $\mathrm{g}$.

However, when the ultrasonic sensors thereon travel to an area where echoes from the real ground level cannot be obtained, the ultrasonic sensors will not produce an effective ground distance value. This is because the echoes received by the ultrasonic sensors are not received from the real ground, but from objects closer to the ultrasonic sensor modules than the ground level, such as branches and leaves of crops. This is especially true if crops got flattened after the storm.

If the error between the current distance and the previously measured distance is less than $-h$ in the recent $N$ sampling periods, the controller determines a virtual ground distance value $g^{\prime}$ by adding the average crop height value $h$ to the top of crop distance value $c$ measured.

$$
g^{\prime}=c+h
$$

Then the virtual ground distance value $g^{\prime}$ is used as the feedback signal of the control system. The value of $N$ will be determined according to the experiment.

b) Design of smoothing algorithm

The surface profile beneath the boom may be thought of as containing a low frequency component due to the slope the spray vehicle is traveling across, also plus a high frequency component due to uneven crop canopy or rough ground. The controller should not respond to these high frequency signals. Therefore, a moving average filter algorithm is designed to smooth the signal.

The DSP controller unit is able to store several periods' worth of data received from the sensors. The controller calculates the moving average once and then update the cumulative moving average with the new values of local crop height or ground height received at each cycle using the following equation:

$$
M A_{i+1}=\frac{D_{i+1}+i M A_{i}}{i+1}
$$

where, $D_{i+1}$ is the new value of distance measured by an ultrasonic sensor; $M A_{i+1}$ is the new cumulative moving average; $M A_{i}$ is the previous value of the cumulative moving average, $i$ is the number of values of local crop height will be stored in the memory. The installation distance of the two ultrasonic sensors is $D$, the angle between the boom and the top of the crop $\theta$ can be calculated using the following formula:

$$
\theta=\arcsin \left(\frac{M A_{L}-M A_{R}}{D}\right)
$$

where, $M A_{L}$ represents the valid distance value between the left boom tip and the target; $M A_{R}$ represents the valid distance value between the right boom tip and the target. c) Data fusion based on optimal weight algorithm

The measured canopy height varies greatly and changes rapidly. The conventional controller only uses the angle measured by the two ultrasonic sensors as the feedback of the controller, it can be found that the chattering phenomena of the spray boom are very obvious during the experiment. In order to solve this problem, an inertial attitude sensor is installed at the center of the center boom frame, and the angle of the ultrasonic sensor is fused with the angle of the center boom measured by the inertial attitude sensor. The specific methods are as follows:

$$
\beta=\theta_{0}+\gamma^{\prime} \Delta t
$$

where, $\gamma$ represents the angular velocity; $\theta_{0}$ represents the boom roll angle measured during the last timer cycle; $\Delta t$ represents the timer interrupt response period.

$Y_{1}$ represents the first derivative of the boom angle measured by two ultrasonic sensors. $Y_{2}$ represents the angular velocity of the boom measured by an inertial sensor. When the absolute value $\left|Y_{1}-Y_{2}\right|$ is greater than $\omega$, using the estimated angular velocity $\hat{\gamma}$ to calculate the boom roll angle $\beta, \hat{\gamma}$ is obtained by multi-sensors data fusion based on optimal weight algorithm, the specific algorithm is as follows

$$
\hat{\gamma}=W_{1} Y_{1}+W_{2} Y_{2}
$$

where, $W_{1}$ and $W_{2}$ are weighted coefficients, and

$$
W_{1}+W_{2}=1
$$

The estimated error variance is

$$
\sigma^{2}=W_{1}^{2} \sigma_{1}^{2}+W_{2}^{2} \sigma_{2}^{2}
$$

where, $\sigma_{1}$ is the measurement error variance of the ultrasonic sensors; $\sigma_{2}$ is the measurement error variance of the inertial measurement sensor.

In order to compute the $W_{j}(j=1,2)$ with the minimum variance $\sigma^{2}$ in Equation (9), we construct auxiliary functions:

$$
f\left(W_{1}, W_{2}, \lambda\right)=\sum_{j=1}^{2} W_{j}^{2} \sigma_{j}^{2}+\lambda\left(\sum_{j=1}^{2} W_{j}-1\right)
$$

The minimum problem of formula (10) under conditional $W_{1}+W_{2}=1$ is reduced to the following conditional extreme value problem

$$
\begin{gathered}
\left\{\begin{array}{l}
\frac{\partial f}{\partial W_{1}}=2 W_{1} \sigma_{1}^{2}+\lambda=0 \\
\frac{\partial f}{\partial W_{2}}=2 W_{2} \sigma_{2}^{2}+\lambda=0
\end{array}\right. \\
W_{1}=\frac{\mu}{\sigma_{1}^{2}}, W_{2}=\frac{\mu}{\sigma_{2}^{2}}, \mu=-\frac{\lambda}{2}
\end{gathered}
$$

It can be derived from Equation (8) and Equation (12)

$$
\mu=\frac{1}{\sum_{i=1}^{N} \frac{1}{\sigma_{i}^{2}}}
$$

Bring Equation (13) into the Equation (12), which can be obtained:

$$
W_{1}=\frac{1}{\sigma_{1}^{2}\left(\frac{1}{\sigma_{1}^{2}}+\frac{1}{\sigma_{2}^{2}}\right)}, W_{2}=\frac{1}{\sigma_{2}^{2}\left(\frac{1}{\sigma_{1}^{2}}+\frac{1}{\sigma_{2}^{2}}\right)}
$$

The optimal weighting factors $W_{1}$ and $W_{2}$ are determined by the error variance $\sigma_{1}$ and $\sigma_{2}$ of each sensor. The variance of measurement errors is calculated from the estimations in the time domain ${ }^{[31]}$

Another noteworthy problem, the angular velocity is obtained by applying the backward difference method to the angle of data fusion. In addition, a second-order Butterworth filter whose cutoff frequency is $5 \mathrm{~Hz}$ is adopted to obtain the angular velocity 
information.

\subsubsection{Controller design}

The conventional PID controller is a linear controller, which constitutes position tracking error $e(t)$ according to the given instruction $\operatorname{rin}(t)$ and the actual output value $y o u(t)$.

$$
e(t)=\operatorname{rin}(t)-y o u(t)
$$

The control law of PID is shown in the following formula

$$
u(t)=k_{p}\left(e(t)+\frac{1}{T_{1}} \int_{0}^{1} e(t) d t+\frac{T_{D} d e(t)}{d \tau}\right)
$$

where, $k_{p}$ is proportional coefficient; $T_{1}$ is integral time constant, and $T_{D}$ is differential time constant; $u(t)$ is the output of the controller.

In order to avoid frequent action of hydraulic cylinders due to small errors, eliminating oscillations that may lead to frequent movements, we set up the response dead band of the PID controller

$$
e(t)=\left\{\begin{array}{cl}
0 & |e(t)| \leq e_{0} \\
e(t) & |e(t)|>e_{0}
\end{array}\right.
$$

where, $e_{0}$ is the threshold value of the dead band. The feedforward control design is based on the idea of compound control, can realize the output fully reproduced input, in order to improve the tracking performance of the system.

The schematic diagram of the feedforward PID control is shown in Figure $8, G_{f}(s)$ represents the transfer function of the feedforward link. The main function of active boom suspension is to make the boom follow the low-frequency variations of the terrain surface, under this condition, the characteristics of the hydraulic servo system can be expressed by velocity integral link, so the velocity feedforward compensation controller is shown as follows

$$
u_{f}(s)=k_{f} \frac{d r i n(t)}{d t}
$$

where, $u_{f}$ is the output of the feedforward compensation link; $s$ is the transform operator; $k_{f}$ is the speed feedforward coefficient.

The output of the velocity feedforward compensation proportional integral control (VFPID) is the sum of the output of the conventional PID control and the velocity feedforward link.

$$
U(t)=u(t)+u_{f}(t)
$$

where, $U(t)$ represents the output of VFPID controller.

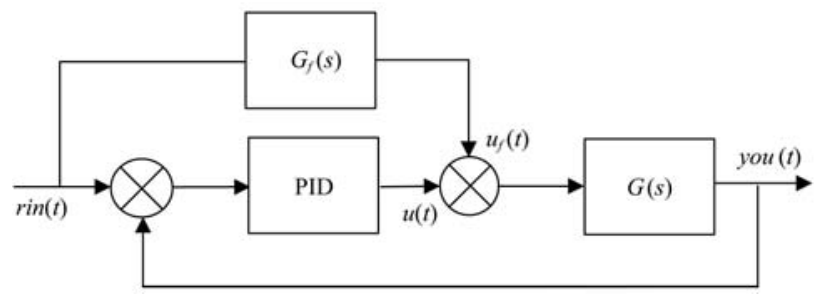

Figure 8 Feedforward PID control

\section{Experiment and result analysis}

\subsection{Experimental setup}

To verify the effectiveness of the designed servo control system, a test bench of active boom suspension system has been set up which is shown in Figure 9. The test bench consists of a six-degrees-of-freedom (6 DOFs) motion simulator (S6DOF, Xingguang Kaiming, Beijing, China), a $28 \mathrm{~m}$ wide spray boom and its pendulum suspension, a hydraulic positioning system, a hydraulic power source, and the designed control systems and human-computer interface.

By mounting a sprayer boom on the platform of 6DOFs motion simulator and reproducing sprayer vehicle vibrations during field operation, it is possible to perform accurate measurements of spray boom movements; the angle between the boom and the ground, two ultrasonic sensors were mounted on the underside of the boom on the left and right sides of the center of gravity of the boom. The 6DOFs motion simulator whose three translations amplitude is $\pm 0.40 \mathrm{~m}$ and three rotations are $\pm 10^{\circ}$, actuation frequency ranges from $0.01 \mathrm{~Hz}$ with an amplitude of $40 \mathrm{~cm}$ to $35 \mathrm{~Hz}$ with an amplitude of $1 \mathrm{~mm}$, and the maximum load quality is $2000 \mathrm{~kg}$.

The control system is realized by using the upper and lower computer system. The program of the hydraulic servo control system is developed on the Code Composer Studio (CCS, Texas Instruments, Texas, USA) software platform. The data acquisition, signal processing and velocity feed-forward PID control algorithm are written in $C$ language. The PC software is programmed with LabVIEW (National Instruments, Texas, USA) to realize the system running state monitoring and experimental data storage function.

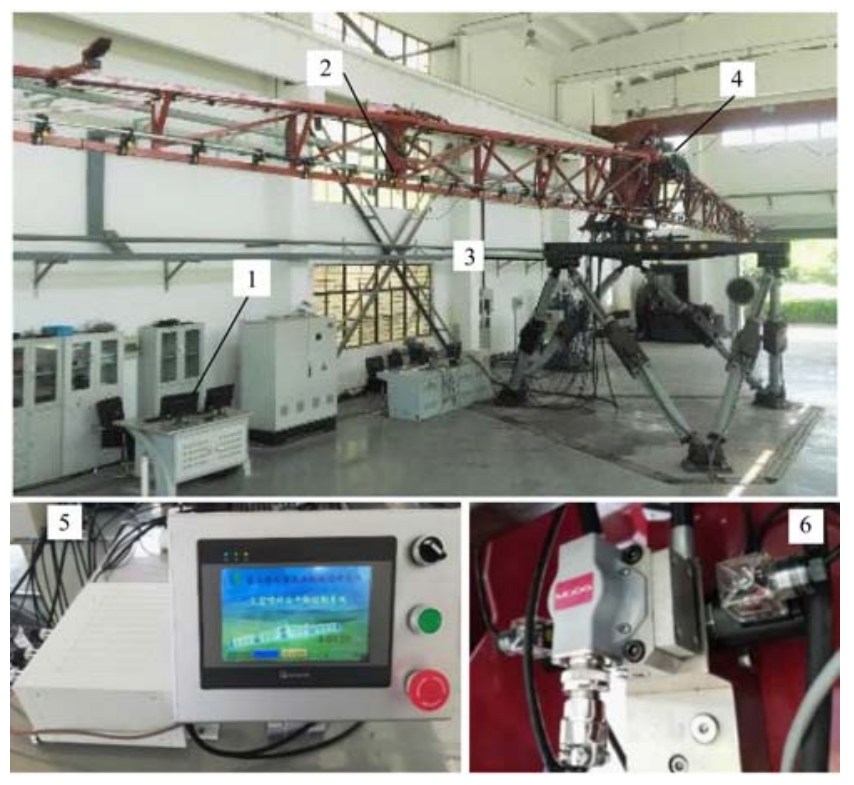

1. Console 2. Proximity sensor 3. Six degrees of freedom motion simulator 4. Spray boom with the pendulum suspension 5. DSP control system and human-computer interface 6. Servo valve

Figure 9 Experimental bench for dynamic characteristics of spray boom suspension

\subsection{Experiments and results}

Parameters of the hydraulic system and the parameters of the controller through calculation and a large number of experimental data. The oil supply pressure of the hydraulic station is $10 \mathrm{MPa}$, the pressure of the oil back is $0.08 \mathrm{MPa}$. Controller gains tuned via trial and error method are $k_{\mathrm{p}}=3.1, k_{\mathrm{i}}=0.6, k_{\mathrm{d}}=0.05, k_{\mathrm{f}}=1.5$, which represent the proportional gain, integrating gain, differential gain and feedforward gain respectively. In the sensor signal processing program, the limiting filter coefficient $N$ is 5 , the smoothing coefficient $i$ is 8 , and the data fusion coefficient $\omega$ is 1.4 . The above control parameters are determined by repeated tests, and if the parameters are enlarged or reduced on the basis of the determined control parameters, which will cause the measurement noise or the high frequency dynamics of the excitation system to make the system unstable.

\subsubsection{Transient response test of a spray boom suspension}

A static experiment to validate the transient response characteristics of the active suspension was carried out. The spray boom was tilted over $6^{\circ}$ and released at that point. The boom rotation is measured by two ultrasonic sensors mounted 
symmetrically with respect to the center of the boom. The motion of the boom till equilibrium for this step input was recorded. This measurement was performed on the passive suspension and active suspension.

The results are shown in Figure 10. The peak time of transient response of active suspension is $1.74 \mathrm{~s}$, and the corresponding peak value is where, $-2.15^{\circ}$, the roll angle of the boom decreases to less than $10 \%$ of the initial value until $3.72 \mathrm{~s}$. In contrast, the peak time of transient response of passive suspension is $2.54 \mathrm{~s}$, and the peak value is $-2.39^{\circ}$, the roll angle of the boom decreases to less than $10 \%$ of the initial value until $5.47 \mathrm{~s}$. Because of the existence of the Coulomb friction, the passive suspension cannot adjust the boom back to the horizontal position, there is an angular deviation of $-0.48^{\circ}$.

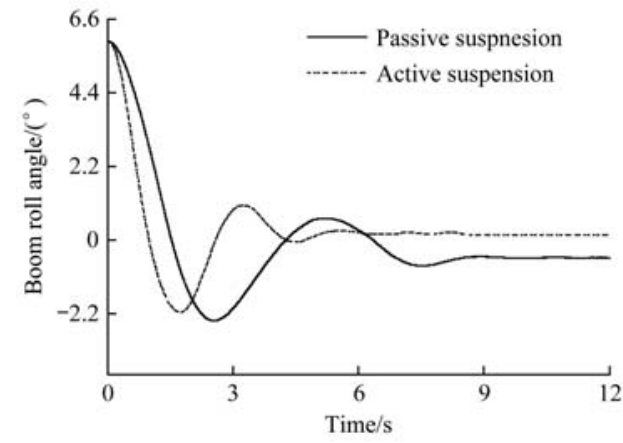

Figure 10 Transient oscillations of the boom when applying a step excitation of $6^{\circ}$

\subsubsection{Sensor signal processing algorithm test}

In order to verify the effectiveness of the proposed limiting filter and anti-shake algorithm, the lifting platform was designed, as shown in Figure 11, it was composed of a bracket, two screw slides, a servo motor, a control cabinet and two range sensors (LTF12UC2LDQ, Banner Engineering Co., Ltd, Minnesota, USA). Two potted plants were placed on the left and right ends of the lifting plate at a distance of about $1.0 \mathrm{~m}$. The platform was pushed through under the ultrasonic sensor from the left side to the right side, and the moving speed of the platform was measured using a ranging sensor. This measurement was performed on two configurations of the current active suspension with and without signal processing algorithm.

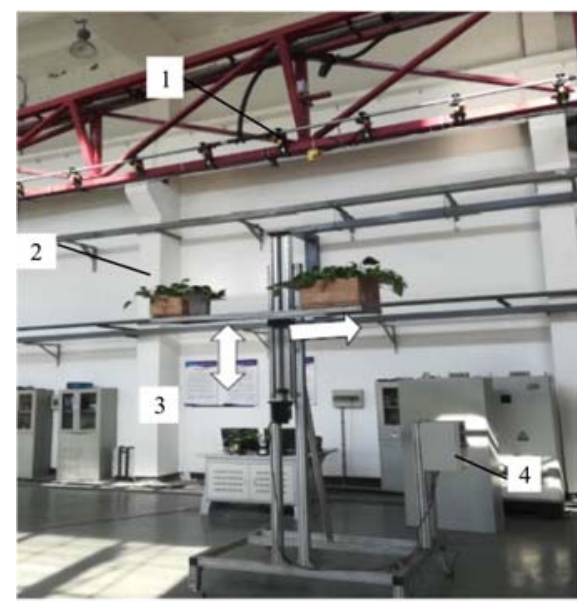

1. Ultrasonic sensor 2. Pot plants 3. Step motor 4. Control cabinet Figure 11 Mobile platform

Pushing the platform from the left side of the ultrasonic sensor to the right side, and the moving speed is $0.5 \mathrm{~m} / \mathrm{s}( \pm 0.1 \mathrm{~m} / \mathrm{s})$. The rolling motion of the boom is tested under the condition of the signal processing algorithm and no signal processing algorithm.
The time history curve of the boom angle is shown in Figure 12. When there is no signal processing algorithm, the response peak of the boom is $-1.39^{\circ}$. After the signal processing algorithm is added, the response peak of the boom is $-0.51^{\circ}$. It can be seen that when signal processing is used, the spray bar does not malfunction due to no crops in the field, thereby increasing the stability of the control system.

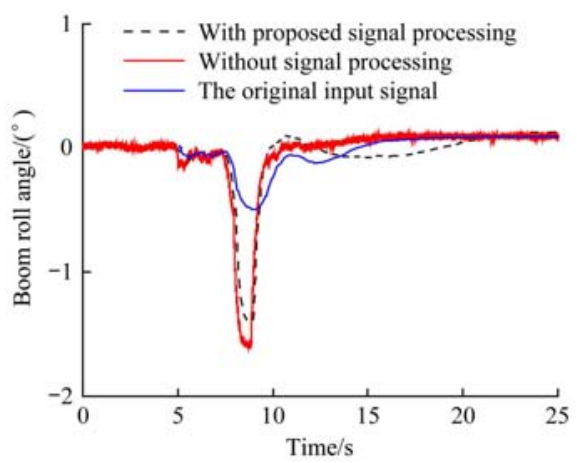

Figure 12 The roll angle of the spray boom

\subsubsection{Sinusoidal interference condition test}

Previous works ${ }^{[18-24]}$ showed that the greatest effect on undesirable spray boom motion is due to the roll motion of spray vehicle, as it travels on undulating terrain. In order to reduce the unevenness in spray deposit, the active suspension should act as a system that isolating the boom from vibrations of spray vehicle. So the boom suspension was tested for a sinusoidal interference condition that represents rolling of the spray vehicle. A sine was used as an input signal, imposing a rolling motion by the motion simulator, with an amplitude of $3^{\circ}$ at a frequency of $0.1 \mathrm{~Hz}$. In this test, the tracking errors of the active suspension and the passive suspension are shown in Figure 13, and their performance indices during the last two cycles are collected in Table 1. Me, $\mu$ and $\sigma$ represent the maximum, average, and standard deviation of the tracking errors respectively.

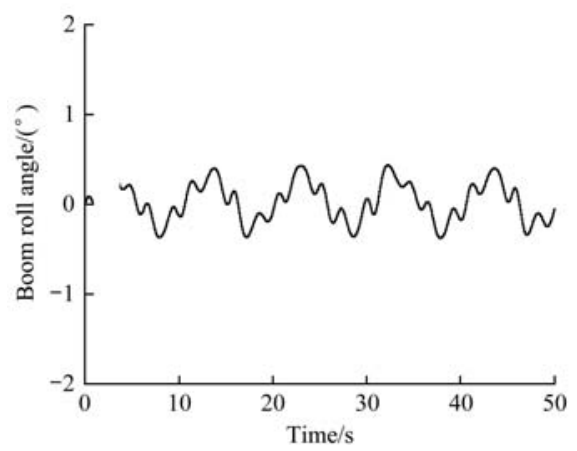

a. Active boom suspension

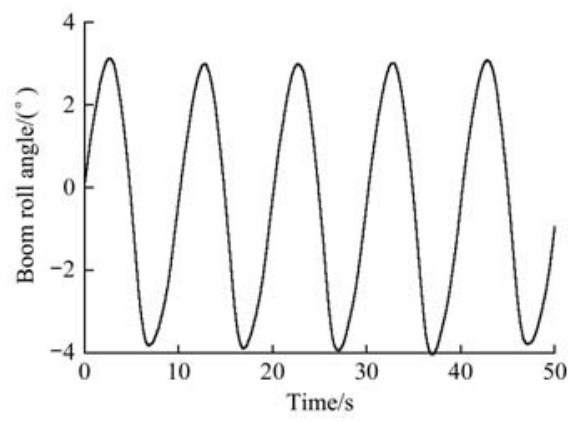

b. Passive suspension

Figure 13 Comparison of the active suspension with the passive suspension for the laboratory experiment using a sine disturbance signal 
Table 1 Statistical indices of boom roll angle

\begin{tabular}{cccc}
\hline Indices & $M \mathrm{e} /\left(^{\circ}\right)$ & $\mu /\left(^{\circ}\right)$ & $\sigma /\left(^{\circ}\right)$ \\
\hline Active suspension & 0.42 & 0.19 & 0.12 \\
Passive suspension & 3.92 & 2.23 & 1.11 \\
\hline
\end{tabular}

The maximum value of the steady-state tracking error of active suspension designed in this paper is $0.42^{\circ}$, and the passive suspension is about $3.92^{\circ}$. These experimental results show that the active suspension achieves better performance since the automatic adjustment of the hydraulic servo position system.

\subsubsection{Sine sweep vibration test}

A swept sine was used as an input signal, imposing a rolling motion on the shaker, with an amplitude of $1^{\circ}$ starting at a frequency of $0.02 \mathrm{~Hz}$ and finishing at $1.0 \mathrm{~Hz}$. The swept sine excites both the resonance and antiresonance frequencies of the suspension. The benefit of a swept sine is that it shows clearly the frequency range in which the control system is active and from which frequency the active damping by the actuator is gradually taken over by passive suspension.

Validation results are shown in Figure 14a and 14b. In both figures the ordinate, $\beta$ presents the angle difference measured by the two ultrasonic devices. $\mathrm{Up}$ to the resonance frequency $0.22 \mathrm{~Hz}$ of the suspension, the controller tries to minimize the inclination angle of boom. Below this resonance frequency, the passive suspension does not react. At the resonance frequency, the active suspension turns out to be more effective than passive suspension, the peak angle of boom for the active system and passive suspension system is $0.72^{\circ}$ and $1.29^{\circ}$ respectively. Beyond the resonance frequency, the active and passive suspension show almost the same damping performance, which is logical because in this region, because the active system is no longer operational by the limiting of output force the hydraulic cylinder.
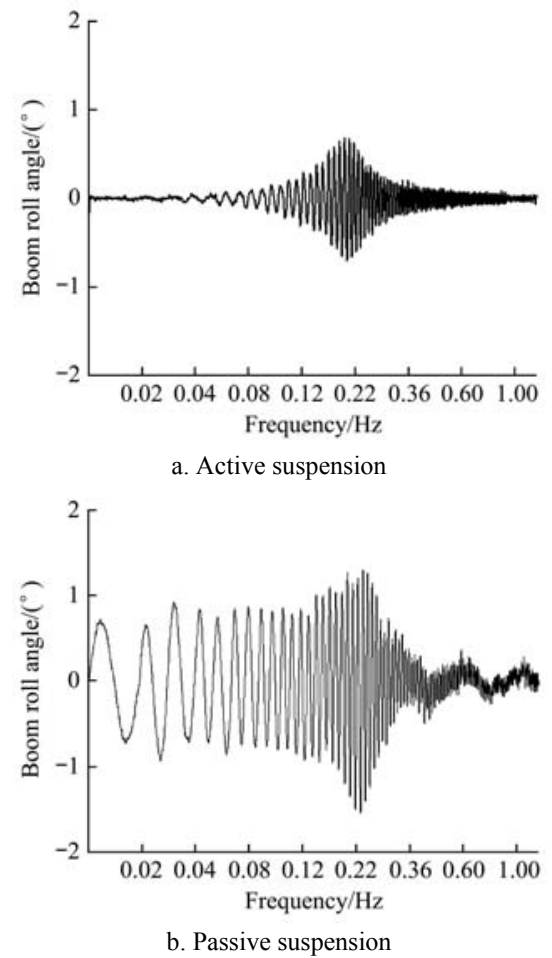

Figure 14 Comparison of the active suspension with the Passive suspension using a swept sine disturbance signal

\subsubsection{Trajectory tracking experiment}

The major role of an active suspension is to maintain the boom parallel to the ground slope or to the crop canopy beneath the boom.
Thus, to control the boom angle, the control system should respond to the low frequency component of the signal which is representative of the ground slope between the boom tips.

To validate the slope following capabilities of the slow active system, a trajectory $\beta_{1 \mathrm{~d}}(t)=3 \cdot \sin (0.1 \cdot \pi t)^{\circ}$ is applied. The Tracking performance of the controller unit can be found in Figure 15a. The tracking error is shown in Figure 15b. The maximum error is $0.57^{\circ}$ in steady state. From the experimental data, we can see that the tracking accuracy of the active suspension to the field slope is greater than $81 \%$. Comparison with sprayer manufactured by John Deere, whose boom was required that the slope of the field should be followed for $70 \%{ }^{[25]}$. The results show that the designed DSP controller can meet the actual use requirements.

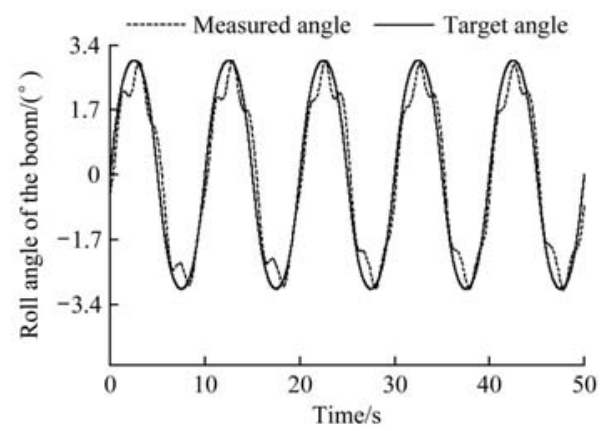

a. Trajectory tracking experiment

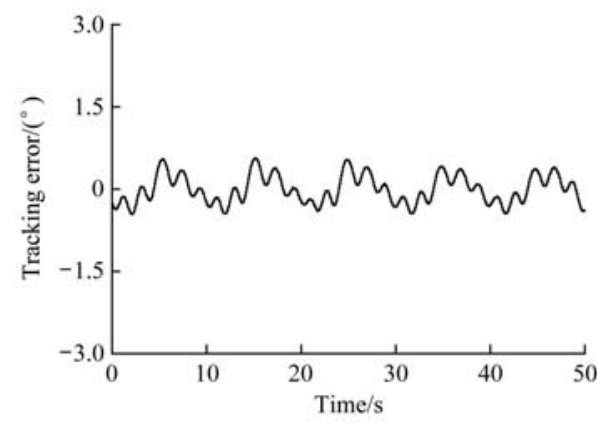

b. Trajectory tracking error

Figure 15 Tracking performance of designed controller

\subsection{Implementation on a real spraying machine}

The active pendulum suspension has been implemented on an operational spraying machine equipped with a boom of $12 \mathrm{~m}$ suspended on the self-propelled sprayer (3WPHS-600A, Shandong Zhonghe Agricultural Equipment Technology Co., Ltd., Yantai, China), as shown in Figure 16. For this suspension, a new controller and electro hydraulic servo system was developed based on the methodology described in Section 3.

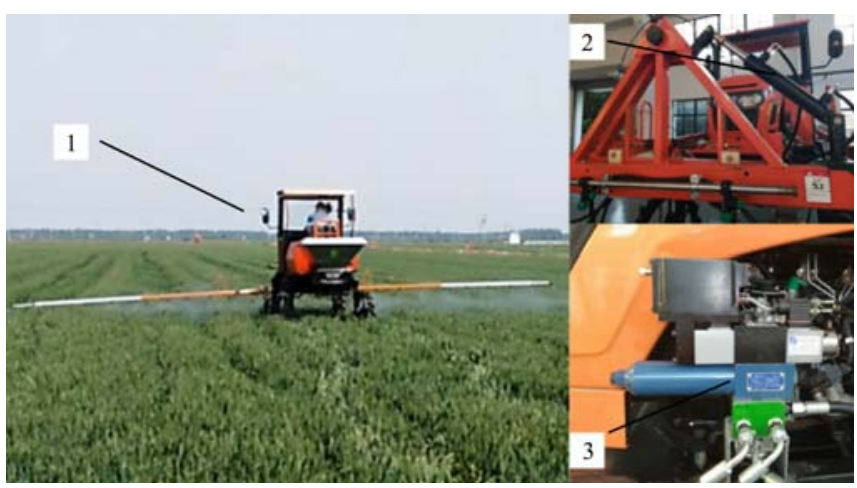

1. Self-propelled sprayer 2. Active pendulum boom suspension 3 . Electro-hydraulic servo control valve

Figure 16 Field test of the sprayer with boom active pendulum suspension 
To validate the slope following capabilities of the active system, a field test was performed at Sanyou Lake Farm, Liuhe District, Nanjing City, Jiangsu Province. The sprayer travels at a speed of $4 \mathrm{~km} / \mathrm{h}$, rolling angle of the boom and chassis were measured by the double GPS aided inertial measurement system (Ellipse-D-G4A2B1, SBG, Cherbourg, France), whose accuracy is $0.05^{\circ}$.

Compare the boom angle with the chassis angle as shown in Figure 17, the statistical Indices of the roll angle for the spray boom and chassis are collected in Table II. The rheology of the soil in the paddy field causes the severe shaking of the sprayer chassis. Under the action of active suspension, the inclination angle of the boom still stays within a very small range. The Standard deviation of roll angle for the boom is $0.40^{\circ}$, the Standard deviation of the chassis angle is $1.04^{\circ}$. It is pointed out that the controller was able to maintain the boom parallel to the crop canopy, and also proves that the suspension system can effectively suppress the disturbance of the chassis.

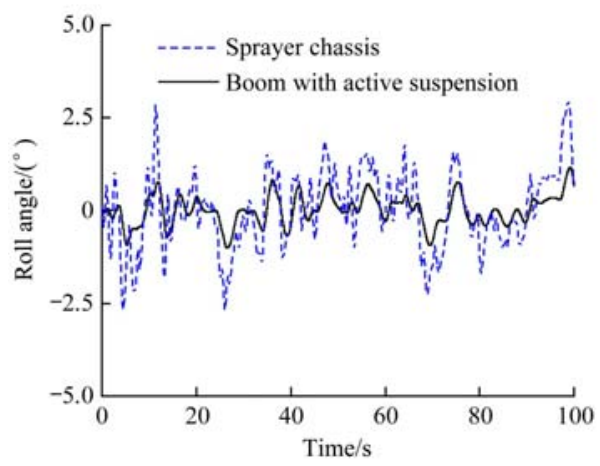

Figure 17 Comparison of boom angle and chassis angle in the field trial

Table 2 Statistical Indices of roll angle for boom and chassis

\begin{tabular}{cccc}
\hline Indices & $M \mathrm{e} /\left(^{\circ}\right)$ & $\mu /\left(^{\circ}\right)$ & $\sigma /\left(^{\circ}\right)$ \\
\hline Roll angle of the boom & 1.17 & 0.31 & 0.40 \\
Roll angle of the chassis & 2.95 & 0.84 & 1.04 \\
\hline
\end{tabular}

\section{Conclusions}

Taking the control system of active suspension as the research object, a high-performance control system hardware circuit and human-computer interaction interface based on DSP28335 chip are designed. Then a special signal processing algorithm is proposed and applied to the motion control of the boom. The main conclusions are as follows:

1) In order to prevent the wrong response of the control system caused by the high frequency component due to uneven crop canopy or rough ground. A special signal processing algorithm is proposed, including the limiting filter, smoothing algorithm and data fusion algorithm based on optimal weight. The experimental comparison shows that the proposed signal processing algorithm significantly improves the reliability of the control system.

2) The effectiveness of the suspension control system was tested using a Stewart six-degree-of-freedom motion simulator. Transient response, sinusoidal tracking and sine sweep test were carried out. Under the step input of $6^{\circ}$, the automatic adjustment time of the active suspension is only $3.72 \mathrm{~s}$. The maximum value of the steady-state tracking error of active suspension is $0.42^{\circ}$, and the passive suspension is about $3.92^{\circ}$ in sinusoidal disturbance test. Results of sine sweep experiments show that the suspension system can track low frequency terrain undulation and attenuate the high-frequency vibration from the chassis by using the electro-hydraulic servo system. Compared with the passive suspension, the low frequency response characteristics of the suspension are significantly improved. At the resonance frequency, the angular amplitude of active suspension and passive suspension are $0.72^{\circ}$ and $1.29^{\circ}$, respectively, and the resonance peak is greatly reduced.

3) The DSP-based controller was implemented on a self-propelled boom sprayer and validated under field conditions, the standard deviation of the roll angle of the boom with active suspension is $0.40^{\circ}$, compared with $1.04^{\circ}$ of the sprayer chassis. The field test results verify the stability and effectiveness of the electro-hydraulic active suspension system. The designed active control system can effectively reduce the effect of ground excitation disturbance during the field application.

\section{Acknowledgements}

We acknowledge that this study was financially supported by the National Key Research and Development Program of China (2017YFD0700905), the National Natural Science Foundation of China (Grant No. 51605236) and the Synergistic Innovation Center of Jiangsu Modern Agricultural Equipment and Technology (4091600002).

\section{[References]}

[1] Harvey F. Growth in crop yields inadequate to feed the world by 2050-research. https://www.theguardian.com/environment/2013/jun/20/ crop- yields-world-population. Accessed on [2017-12-13].

[2] $\mathrm{He} X \mathrm{~K}$. Improving severe draggling actuality of plant protection machinery and its application techniques. Transactions of the CSAE, 2004; 20(1): 13-15. (in Chinese)

[3] Herbst A, Osteroth H, Fleer W, Stendel H. A method for testing automatic spray boom height control systems. ASABE Annual International Meeting, New Orleans: ASABE, 2015; pp.11-17.

[4] Jia W D, Zhang L J, Yan M D, Xue X Y. Current situation and development trend of boom sprayer. Journal of Chinese Agricultural Mechanization, 2013; 34(4): 19-22. (in Chinese)

[5] Langenakens J J, Clijmans L, Ramon H. The effects of vertical sprayer boom movements on the uniformity of spray distribution. Journal of agricultural engineering research, 1999; 74(3): 281-291.

[6] Wilkerson J B, Womac A R, Hart W E, Hong Y J. Sprayer boom instrumentation for field use. Transactions of the ASAE, 2004; 47(3): 659-666.

[7] Batte M T, Ehsani M R. The economics of precision guidance with auto-boom control for farmer-owned agricultural sprayers. Computers and Electronics in Agriculture, 2006; 53(1): 28-44.

[8] Iyer R M, Wills B. Factors determining the design of tractor-mounted sprayer booms - sprayer nozzle characteristics. Journal of Agricultural Engineering Research, 1978; 23(1): 37-43.

[9] Lardoux Y, Sinfort C, Enfält P, Sevila F. Test method for boom suspension influence on spray distribution, part I: Experimental study of pesticide application under a moving boom. Biosystems Engineering, 2007; 96(1): 29-39.

[10] Lardoux Y, Sinfort C, Enfält P, Miralles A, Sevila F. Test method for boom suspension influence on spray distribution, part II: Validation and use of a spray distribution model. Biosystems Engineering, 2007; 96(2): $161-168$.

[11] Balsari P, Gil E, Marucco P. Field-crop-sprayer potential drift measured using test bench: Effects of boom height and nozzle type. Biosystems Engineering, 2017; 154: 3-13.

[12] Gil E, Balsari P, Gallart M, Llorens J, Marucco P. Determination of drift potential of different flat fan nozzles on a boom sprayer using a test bench. Crop Protection, 2014; 56: 58-68.

[13] Gil E, Gallart M, Balsari P, Marucco P. Influence of wind velocity and wind direction on measurements of spray drift potential of boom sprayers using drift test bench. Agricultural and Forest Meteorology, 2015; 202: 
94-101.

[14] Ramon H, Baerdemaeker J D. Spray boom motions and spray distribution: part 1, tion of a mathematical relation. Journal of Agricultural Engineering Research, 1997; 66(1): 23-29.

[15] Ramon H, Missotten B, Baerdemaeker J D. Spray boom motions and spray distribution, Part 2: Experimental validation of the mathematical relation and simulation results. Journal of Agricultural Engineering Research, 1997; 66(1): 31-39.

[16] O'sullivan J A. Simulation of the behavior of a spray boom with an active and passive pendulum suspension. Journal of Agricultural Engineering Research, 1986; 35(3): 157-73.

[17] Pontelli C O, Mucheroni M F, Balthazar J M. Comparison between two types of passive boom suspension under simulated conditions of track test. Engenharia Agrícola, 2010; 30(4): 761-775.

[18] Langenakens J J, Clijmans L, Ramon H. The Effects of Vertical Sprayer Boom Movements on the Uniformity of Spray Distribution. Journal of Agricultural Engineering Research, 1999; 74(3): 281-291.

[19] Cui L F, Xue X Y, Ding S M, Qiao B Y, Le F X. Analysis and test of dynamic characteristics of large spraying boom and pendulum suspension damping system. Transactions of the CSAE, 2017; 33(9): 61-68. (in Chinese)

[20] Cui L F, Mao H P, Xue X Y, Ding S M, Qiao B Y. Optimized design and test for a pendulum suspension of the crop spray boom in dynamic conditions based on a six DOF motion simulator. Int $\mathrm{J}$ Agric \& Biol Eng, 2018; 11(3): 76-85.

[21] Cui L F, Xue X Y, Ding S M, Gu W, Chen C, Le F X. Modeling and simulation of dynamic behavior of large spray boom with active and passive pendulum suspension. Transactions of the CSAM, 2017; 48(2): 82-90. (in Chinese).

[22] Marchant J A, Frost A R. Simulation of the performance of state feedback controllers for an active spray boom suspension. Journal of Agricultural Engineering Research, 1989; 43: 77-91.
[23] Deprez K, Anthonis J, Ramon H, Hvan B. Development of a slow active suspension for stabilizing the roll of spray booms, Part 1: Hybrid modelling. Biosystems Engineering, 2002; 81(2): 185-191.

[24] Deprez K, Anthonis J, Ramon H. System for vertical boom corrections on hilly fields. Journal of Sound \& Vibration, 2003; 266(3): 613-624.

[25] Anthonis J, Audenaert J, Ramon H. Design optimization for the vertical suspension of a crop sprayer boom. Biosystems Engineering, 2005; 90(2) $153-160$.

[26] Tahmasebi M, Mailah M, Gohari M, Rahman R A. Vibration suppression of sprayer boom structure using active torque control and iterative learning. Part 1: Modeling and control via simulation. Journal of Vibration \& Control, 2017; 2: 177-186.

[27] Tahmasebi M, Gohari M, Mailah M. Vibration suppression of sprayer boom structure using active torque control and iterative learning. Part II: Experimental implementation. Journal of Vibration \& Control, 2017; 10: 107-125.

[28] Chen W F, Qiu B J, Yang N, Yin L. spray boom position control system based on ultrasonic sensors. Journal of Agricultural Mechanization Research, 2013; 3: 84-87. (in Chinese)

[29] Wang S L, Zhao C J, Wang X. Design and experiments on boom height adjusting system. Journal of Agricultural Mechanization Research, 2014; 8: 161-164. (in Chinese)

[30] Pan F C, Deng F D, Wen H J. Design and experiments on boom height automatic adjusting system. Journal of Agricultural Mechanization Research, 2017; 11: 107-111. (in Chinese)

[31] Satow T, Miyamoto K, Matsuda K. Control of spraying height with ultrasonic sensor for boom sprayer. (Part 2) development of automatic control device for spraying height. Journal of the Japanese Society of Agricultural Machinery, 2010; 56(2): 59-67.

[32] Gao S S, Zhong Y M, Shirinzadeh B. Random weighting estimation for fusion of multi-dimensional position data. Information Sciences, 2010; 180(24): 4999-5007. 